\title{
A】l102 b】6394
}

NATL INST OF STANDARDS \& TECH R.I.C. |

A11102616394

QC100.U5753 NO.

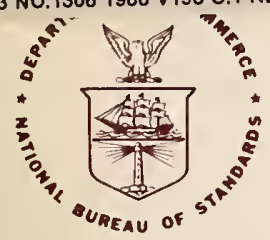

\section{NBS TECHNICAL NOTE 1306}

U.S. DEPARTMENT OF COMMERCE / National Bureau of Standards

\section{Displacement Errors in Antenna Near-Field Measurements and Their Effect on the Far Field}

Lorant A. Muth

QC

100

- 45753

No. 1306

1986

c. 2 
he National Bureau of Standards ${ }^{1}$ was established by an act of Congress on March 3, 1901. The Bureau's overall goal is to strengthen and advance the nation's science and technology and facilitate their effective application for public benefit. To this end, the Bureau conducts research and provides: (1) a basis for the nation's physical measurement system, (2) scientific and technological services for industry and government, (3) a technical basis for equity in trade, and (4) technical services to promote public safety. The Bureau's technical work is performed by the National Measurement Laboratory, the National Engineering Laboratory, the Institute for Computer Sciences and Technology, and the Institute for Materials Science and Engineering.

\section{The National Measurement Laboratory}

Provides the national system of physical and chemical measurement; coordinates the system with measurement systems of other nations and furnishes essential services leading to accurate and uniform physical and chemical measurement throughout the Nation's scientific community, industry, and commerce; provides advisory and research services to other Government agencies; conducts physical and chemical research; develops, produces, and distributes Standard Reference Materials; and provides calibration services. The Laboratory consists of the following centers:
- Basic Standards ${ }^{2}$

- Radiation Research

- Chemical Physics

- Analytical Chemistry

\section{The National Engineering Laboratory}

Provides technology and technical services to the public and private sectors 10 address national needs and to solve national problems; conducts research in engineering and applied science in support of these efforts; builds and maintains competence in the necessary disciplines required to carry out this research and technical service; develops engineering data and measurement capabilities; provides engineering measurement traceability services; develops test methods and proposes engineering standards and code changes; develops and proposes new engineering practices; and develops and improves mechanisms to transfer results of its research to the ultimate user. The Laboratory consists of the following centers:
- Applied Mathematics

- Electronics and Electrical Engineering ${ }^{2}$

- Manufacturing Engineering

- Building Technology

- Fire Research

- Chemical Engineering?

\section{The Institute for Computer Sciences and Technology}

Conducts research and provides scientific and technical services to aid Federal agencies in the selection, acquisition, application, and use of computer technology to improve effectiveness and economy in Government operations in accordance with Public Law 89-306 (40 U.S.C. 759), relevant Executive Orders, and other directives; carries out this mission by managing the Federal lnformation Processing Standards Program, developing Federal ADP slandards guidelines, and managing Federal participalion in ADP voluntary standardization activities; provides scientific and technological advisory services and assistance to Federal agencies; and provides the technical foundation for computer-related policies of the Federal Government. The Institute consists of the following centers:
- Programming Science and Technology

- Computer Sysiems Engineering

\section{The Institute for Materials Science and Engineering}

Conducts research and provides measurements, data, standards, reference materials, quantitative understanding and other technical information fundamental to the processing, structure, properties and performance of materials; addresses the scientific basis for new advanced materials technologies; plans research around cross-country scientific themes such as nondestructive evaluation and phase diagram development; oversees Bureau-wide technical programs in nuclear reactor radiation research and nondestructive evaluation; and broadly disseminates generic technical information resulting from its programs. The Institute consists of the following Divisions:

\section{- Ceramics}

- Fracture and Deformation ${ }^{3}$

- Polymers

- Metallurgy

- Reactor Radiation 


\title{
Displacement Errors in Antenna Near-Field Measurements and Their Effect on the Far Field
}

\author{
Lorant A. Muth
}

Electromagnetic Fields Division

Center for Electronics and Electrical Engineering

National Engineering Laboratory

National Bureau of Standards

Boulder, Colorado 80303

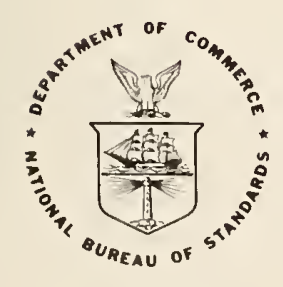

U.S. DEPARTMENT OF COMMERCE, Malcolm Baldrige, Secretary NATIONAL BUREAU OF STANDARDS, Ernest Ambler, Director 
National Bureau of Standards Technical Note 1306

Natl. Bur. Stand. (U.S.), Tech Note 1306, 36 pages (Oct. 1986) CODEN:NBTNAE

U.S. GOVERNMENT PRINTING OFFICE

WASHINGTON: 1986 


\section{CONTENTS}

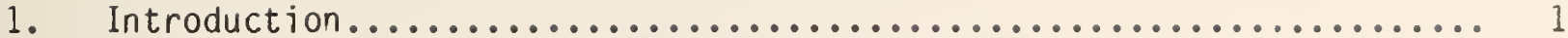

2. General Mathernatical Statement of the Problem.................. 2

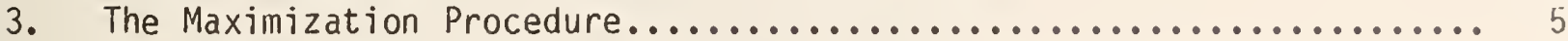

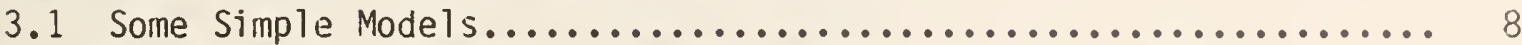

3.2 Maximization of General Complex Near-Field Error Integral ..... 10

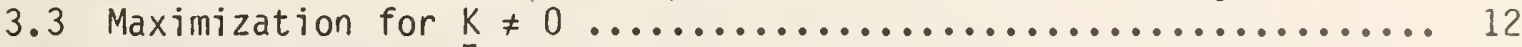

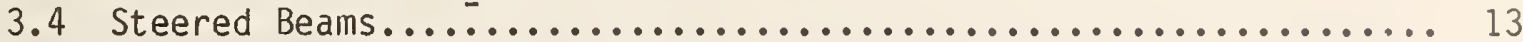

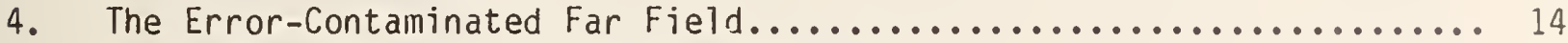

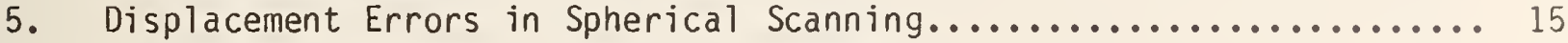

5.1 A Simple Model in Spherical Geometry.................... 19

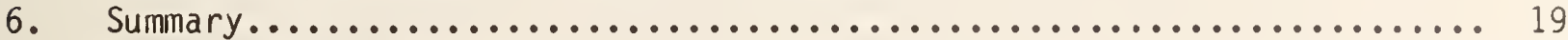

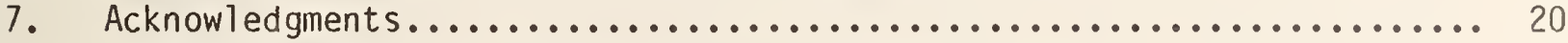

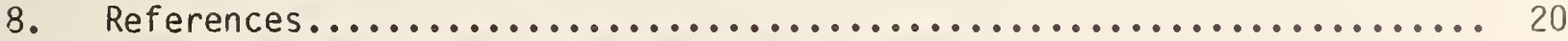

Appendix A Maximization of General Complex Near-Field Error

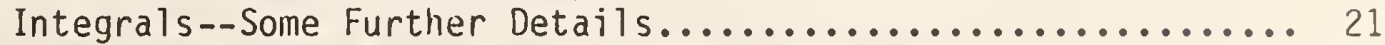

Appendix B The Maximization Procedure for Steered Beams.............. 23

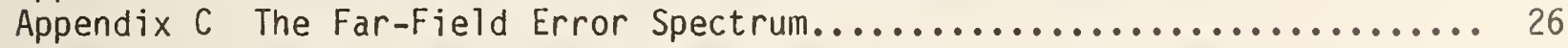

Appendix D Radial Displacement Errors in Spherical Scanning............ 29 

Displacement Errors in Antenna Near-Field Measurements and Their Effect on the Far Field

by

Lorant A. Muth

The effects of probe displacement errors in the near-field measurement procedure on the far-field spectrum are studied. Expressions are derived for the displacement error functions that maxiinize the fractional error in the spectrum both for the on-axis and off-axis directions. Planar $x-y$ and $z$-displacement errors are studied first and, consequently, the results are generalized to errors in spherical scanning. Some simple near-field models are used to obtain order of magnitude estimates for the fractional error as a function of relevant scale lengths of the near field, defined as the lengths over which significant variations occur.

Key words: error maximization; far-field spectrum; near fields; off-axis fractional errors; on-axis fractional errors; planar scanning; probe displacement errors; spherical scanning.

1. Introduction

The unavoidable errors in the probe's position while scanning the near field of an antenna show up inevitably in the far field of the antenna being measured. As is well known [1], the far field of an antenna is obtained by taking the Fourier transform of the antenna's planar near field and performing additional algebraic manipulations to remove the effect of the receiving characteristics of the probe, a procedure known as probe correction. In evaluating the accuracy of a near-field range--planar, cylindrical or spherical-obvious and natural questions arise: 1) what systematic position errors will lead to maximum far-field errors; 2) what is the dependence of this maximum far field error function on the wave number $\underline{k}$, whose magnitude is constant; and, more generally, 3) what is the exact error-contaminated far field of an antenna if its exact near field and an arbitrary probe displacement error function are known?

Two previous studies raised the first two questions above $[2,3]$ and treated them in the context of planar scanning. This paper re-examines the above question in the planar context from a slightly different point of view, with the intent to achieve enough generality in the mathematical formalism so 
that the analysis can be extended to study position errors in cylindrical and spherical scanning procedures. Some error expressions are derived in spherical geometry which can serve as the basis for computer simulation. Similar simulations in the planar case have been discussed in [3]. A general expression that answers the third question is also derived.

To accomplish the objectives of the paper, expressions for maximum systematic errors in all geometries have to be derived. First, simple general mathematical arguments are used to get the structure and the relevant parameters that appear in the fractional error expressions; then a rigorous procedure for maximization of error is outlined for on-axis errors in real near fields. This simplified special case is considered first for a mathematical reason: the error expressions for realistic (complex) near fields for on- or off-axis directions in k-space can be obtained using the procedure worked out for the simplified on-axis case if a straightforward additional procedure is incorporated. Once this procedure is worked out, all special complicated cases such as steered beams and all errors in spherical geometry can be treated.

2. General Mathematical Statement of the Problem

We can derive general expressions for the fractional error in the spectrum of the near field $B(\underline{x})$ due to arbitrary position errors. Here $\underline{x}$ is an arbitrary three-dimensional position vector. If the real function $\underline{\delta x}(\underline{x})$ is the error in position of the probe at $\underline{x}$, then the near field measured is $B(\underline{x}+\delta \underline{x}(\underline{x}))$. The fractional error in the spectrum $D(\underline{k})$ due to position errors is then

$$
\frac{\Delta D(\underline{k})}{D(\underline{k})}=\frac{\int\{B(\underline{x}+\delta \underline{x}(\underline{x}))-B(\underline{x})\} e^{-i \underline{k} \cdot \underline{x}} d^{2} x}{\int B(\underline{x}) e^{-i \underline{k} \cdot \underline{x}} d^{2} x}
$$

where the integration is over the finite scan plane. The use of a finite plane of integration is an approximation in the denominator, but is exact in the numerator, since errors occur only at points where measurements are taken. In the above expression we also have $\underline{k}=(\underline{K}, \pm \gamma)$, where $\underline{k}=\left(k_{x}, k_{y}\right)$, $k_{z}= \pm \gamma$ and $|k|=\frac{2 \pi}{\lambda}=$ constant for lossless media. We now seek that real 
function $\delta \underline{x}(\underline{x})$ that maximizes the fractional error in a given direction in $k-$ space. The numerator in eq (1) will have a maximum for a finite $\delta \underline{x}(\underline{x})$ only if $\delta \underline{x}(\underline{x})$ is subject to the constraint

$$
\frac{1}{A} \int|\delta \underline{x}|^{2} d^{2} x=\sigma^{2}
$$

where $\sigma=$ constant, $A$ is the total scan area. Expressions (1) and (2) then define a variational problem wherein the function $\underline{\delta} \underline{x}(\underline{x})$ that maximizes eq (1) can be found with the use of the functional derivative [4]. This simple procedure will be indicated below for the one-dimensional case. Physically, the constraint in eq (2) merely restricts the error functions to be considered to a constant RMS value. Strictly speaking, the variational problem has to be formulated for either the real or imaginary part of eq (1) separately. To find the maximum of the absolute value of the fractional error, a slightly modified procedure has to be followed.

One can write down very general expressions for the maximum fractional error in eq (1) without having to specify $\delta \underline{x}(\underline{x})$. By the use of the mean value theorem for real functions [5] and for $\underline{K}=0$ (on axis), one can write (assuming that any $z$-dependence is specified as a function of $x$ and $y$, and suppressing the $k_{z}= \pm \gamma$ dependence)

$$
\mu \equiv \frac{\Delta D(0)}{D(0)}=\frac{B(\underline{\hat{x}}+\delta \underline{x}(\underline{\hat{x}}))-B(\underline{\hat{x}})}{B(\underline{\bar{x}})}
$$

where $\hat{x}$ and $\underline{\bar{x}}$ are some points on the scan plane and $B(\underline{x})$ is the average of the near-field measurements. We have assumed here that $B(\underline{x})$ is essentially a real function (complex phase is allowed), since the mean value theorem cannot be applied to complex functions directly [6].* We can approximate eq (3) as

\footnotetext{
* To reiterate, these simplifying assumptions are made here in order to develop an understanding of the relevant parameters and the structure of the error expressions sought. It is not true that this simple example is studied because more cornplicated cases cannot be treated. As will be seen below, the error analysis of any special case that is more general than the one considered here follows simply from the considerations in this section and the next and the additional procedure outlined in section 3.2 .
} 


$$
\frac{\Delta D(0)}{D(0)}=\frac{|\nabla B||\delta \underline{x}|}{B(\underline{x})}
$$

which in the one-dimensional case is (prime denotes differentiation)

$$
\frac{\Delta D(0)}{D(0)}=\frac{B^{\prime}(\hat{x}) \delta \underline{x}(\hat{x})}{B(\bar{x})} \leqslant \frac{B^{\prime}{ }_{\max } \delta x_{\max }}{B(\bar{x})} .
$$

As will be seen below, in a first order approximation a properly normalized displacement-error function has a maximum proportional to $\sigma$, hence (using a as constant of proportionality),

$$
\mu_{1} \equiv \frac{\Delta D(0)}{D(0)} \leqslant \alpha \sigma \frac{B^{\prime}{ }^{\prime} \max }{B(\bar{x})}=c \frac{\sigma}{\ell}
$$

where $c$ is some constant of order unity, and $\ell$ is scale length of significant variation in the near field. In eq (5a) and below, the subscript on $\mu$ indicates the order of approximation. The exact value of $c$ can be obtained only from either an actual near field or a model of it. An order-of-magnitude estimate for $c$ can be obtained as follows: $B^{\prime}{ }_{\max } \approx B_{\max } / \ell, B(\bar{x}) \approx B_{\max } / 2$ and assuming $\alpha \approx 1$, one obtains $c \approx 2$. As will be seen below, this is, indeed, a good estimate. (Equation (5a) agrees in form with eq (63a) in [3]).

The scale length $\ell$ represents variations in the near field $B(x)$ either parallel or perpendicular to $\underline{k}_{0}$, the wave vector of interest. In case $\delta \underline{x}_{-0}$, then $e^{-1} \approx\left|k_{0}\right|=\frac{2 \pi}{\lambda}$, as can be verified from the simple model of a plane wave of constant amplitude propagating in the direction $\delta \underline{x}$. Variations in the amplitude of the near field orthogonal to $\delta \underline{x}$ will be reflected in the constant in eq $(5 a)$, in this case denoted by $c_{\|}$. For $\delta \underline{x} \perp \underline{k}$, $\ell$ represents the scale length in the variation of the near-field profile along surfaces of constant phase. The constant in this case will be denoted by $c_{\perp}$. Hence, we can write

$$
\mu_{1}^{(11)}<c_{\|} k \sigma_{\|}
$$

and

$$
\mu_{1}^{(1)}<c_{1} \frac{\sigma_{1}}{\ell} .
$$


In the case of an on-axis beam $(\underline{K}=0)$ eq $(5 b)$ represents the upper bound error resulting from z-displacement errors (out of the scan plane), and eq $(5 c)$ represents the upper bound resulting from displacement errors in the scan plane. The ratio

$$
\frac{\mu(1)}{\mu} \approx \frac{\lambda}{l} \ll 1
$$

in general.

Two alternative expressions to eq (4) can be shown to be, using $\beta$ for the unit amplitude near field and denoting derivatives by ',

$$
\left|\frac{\Delta D(0)}{D(0)}\right| \leqslant \sigma \frac{\beta^{\prime} \text { max }}{\langle\beta\rangle} \frac{\beta^{\prime} \text { max }}{\left\langle\beta^{\prime 2}\right\rangle^{1 / 2}},
$$

and

$$
\left|\frac{\Delta D(0)}{D(0)}\right|^{2} \leqslant \sigma^{2} \frac{\left\langle\left[\beta^{\prime}(x)\right]^{2}\right\rangle}{\langle\beta(x)\rangle^{2}},
$$

where the \langle\rangle implies the average. These expressions will be derived in the next section. Again, the derivatives in eqs (6) and (7) represent directions either parallel or orthogonal to $\underline{k}$.

In eqs (5) through (7) it has been assumed that the near field is real. If the fractional error in directions other than $\underline{K}=0$ is desired or if the near field is complex (i.e., for any real antennas, steered beams or electrically small antennas), the derivations of scale-length expressions are slightly more complicated, but fundamentally present no great difficulties.

\section{The Maximization Procedure}

To solve the variational problem as stated in eqs (1) and (2) in complete generality, we have to proceed in steps. First, we solve the simplified problem, where the near field is a real function and the wave vector in the exponential vanishes. Then we seek to maximize (in 1-D) the integral

$$
I=\int_{L}[B(x+\delta x)-B(x)] d x,
$$


subject to the constraint

$$
\frac{1}{L} \int_{L}(\delta x)^{2} d x=1
$$

where $L$ is the interval of interest. We use the Lagrange multiplier $n$ [4] to maximize

$$
\hat{I}=\int[B(x+\delta x)-B(x)] d x-\eta \int(\delta x)^{2} d x
$$

with respect to $\delta x$. Thus,

$$
\frac{\delta \hat{I}}{\delta(\delta x)}=\int_{L}\left[B^{\prime}(x+\delta x)-2 n \delta x\right] d x=0
$$

or

$$
\delta x=\frac{1}{2 \eta} B^{\prime}(x+\delta x) .
$$

Equation (10c) is an implicit statement specifying $\delta x(x)$ that will maximize the integral in eq (8). To obtain $\delta x(x)$ explicitly, eq (10c) is expanded in a Taylor series and the constant $n$ is obtained from eq (9). The conditions for first- and higher-order approximation values can thus be worked out. If we want the displacement-error function to satisfy

$$
\frac{1}{L} \int_{L}(\delta x)^{2} d x=\sigma_{x}^{2} \neq 1
$$

instead of condition (9), we merely have to multiply $\delta x$ in eq (9) by $\sigma_{x}$. Thus there is no loss of generality in using eq (11), as eq (9) is a special case of eq (11).

The Taylor series expansion of eq (10c) is

$$
\delta x=\frac{1}{2 n}\left\{B^{\prime}(x)+B^{\prime \prime}(x) \delta x+\frac{1}{2 !} B^{\prime \prime}(x)(\delta x)^{2}+\cdots\right\}
$$

For a first-order expansion in $\delta x$ the condition

$$
\left|\frac{1}{2 !} \frac{B^{\prime \prime \prime}(x)}{B^{\prime \prime}(x)}(\delta x)\right| \ll 1
$$


must hold, and similarly for higher-order terms. This implies

$$
\delta x=\frac{B^{\prime}(x)}{2 n-B^{\prime \prime}(x)}
$$

where $n$ is determined by eq (11). If we further assume that

$$
B^{\prime \prime}(x)<2 n
$$

then from eqs (13) and (11)

$$
\frac{1}{4 n^{2} L} \int_{L}\left[B^{\prime}(x)\right]^{2} d x=\sigma_{x}^{2}
$$

and

$$
\delta x \stackrel{\simeq}{=} \frac{B^{\prime}(x)}{\left\langle B^{\prime} 2\right\rangle} 1 / 2
$$

satisfies eq (11). Consistent with the first-order approximation developed here, the integral in eq (8) is approximated as

with

$$
I \cong \int_{L} B^{\prime}(x) \delta x d x
$$

$$
\left|\frac{1}{2 !} \frac{B^{\prime \prime}(x)}{B^{\prime}(x)} \delta x\right| \ll 1
$$

and similarly for higher-order terms. Using eq (15), eq (12) becomes

$$
\left|-\frac{x}{2} \frac{B^{\prime \prime \prime}(x)}{B^{\prime \prime}(x)} \frac{B^{\prime}(x)}{\left\langle B^{\prime} 2\right\rangle} 1 / 2\right| \ll 1
$$

and eqs (14) and (18) are consistently satisfied by

$$
\left|\sigma_{x} \frac{B^{\prime \prime}(x)}{\left\langle B^{\prime} 2\right\rangle} 1 / 2\right| \ll 1 .
$$

It is easy to see that eqs (19a) and (19b) can be satisfied for a small enough $\sigma_{x}$. Special attention must be given to points where $B^{\prime \prime}(x)=0$, but we will not address that here.

The maximum of eq (17) is obtdined using eq (15) 


$$
I \cong \sigma_{X} L\langle B \cdot 2\rangle / 2
$$

and the fractional error in eq (1) can be written as in eq (7)

$$
\mu_{1}^{2}=\left|\frac{\Delta D(0)}{D(0)}\right|^{2} \leqslant \sigma_{x}^{2} \frac{\left\langle B^{\prime} 2\right\rangle}{\langle B(x)\rangle^{2}}=\sigma_{x}^{2} \frac{\left\langle\beta^{\prime 2}\right\rangle}{\langle\beta(x)\rangle^{2}}
$$

where $\beta(x)$ is the unit-amplitude near field. Higher-order approximation schemes to solve eqs ( 8 ) and (10) consistently can be worked out, but this will not be done here, since the unwieldy algebraic manipulations lead to no new results. In eqs (1) and (2) a second-order expansion of the integral in eq (8) has been found useful, i.e., the maximization of

$$
\int\left\{B^{\prime}(x) \delta x+\frac{1}{2 !} B^{\prime \prime}(x)(\delta x)^{2}\right\} d x
$$

has been sought. We can use the first-order expression (15) to get

$$
\mu_{2} \leqslant \frac{1}{\langle\beta\rangle}\left[\sigma_{X}\left\langle\beta^{\prime 2}\right\rangle^{1 / 2}+\frac{1}{2 !} \sigma_{X}^{2} \frac{\left\langle\beta^{\prime \prime} \beta^{\prime 2}\right\rangle}{\left\langle\beta^{\prime 2}\right\rangle}\right] \text {. }
$$

The more exact expression for $\mu_{2}$ using a second-order expansion in eq (10) would result in a much more complicated form.

Since $\Delta D \equiv D_{e}-D_{0}$, where $D_{e}$ is the error-contaminated spectrum, one can write $D_{e}=D_{0}\left(1+\mu_{1}\right)$ or

$$
R(d B) \equiv 20 \log _{10}\left(\frac{D}{D_{0}}\right) \approx 8.7 \mu_{1} .
$$

This result together with eq (21a) can be compared to that given in [3].

Before proceeding to maximize eq (8) for complex near fields $B(x)$ or for $\underline{k} \neq 0$ (off axis), a few elementary near-field models will be used to exhibit some explicit results for the fractional error $\mu$.

\subsection{Some Simple Models}

Two basic models that incorporate the most essential features of near fields will be used in expressions (21) and (22). These are a triangle and 


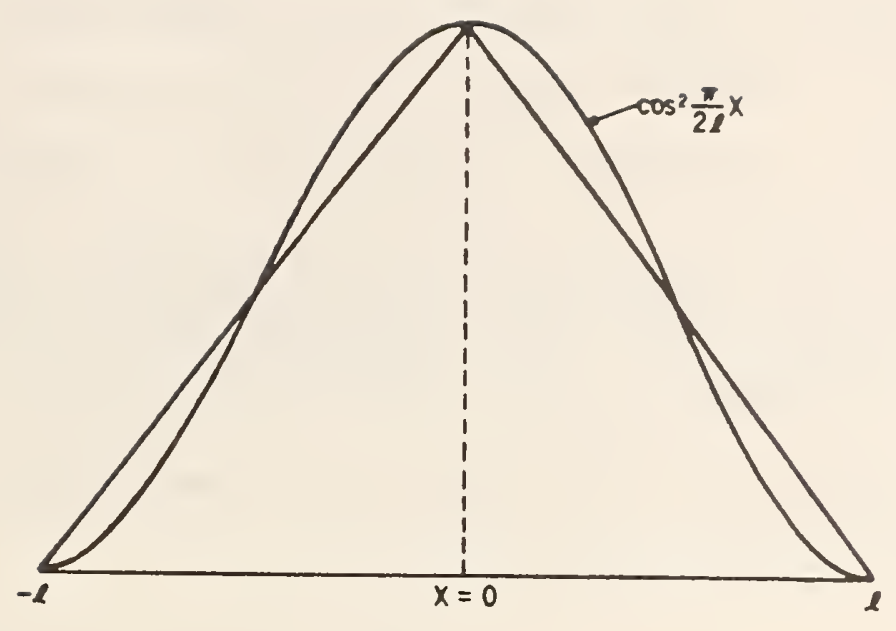

Figure 1. Simple models for the near field to calculate the effects of probe displacement errors

a $\cos ^{2} \alpha x$, where $\alpha=\frac{\pi}{2 l}$. These are illustrated in figure 1 . Both of these models have a scale length $\ell$. The model independence of the maximization process can be surmised by deriving results for both of these models. For the triangle $\left|B^{\prime}\right|=\frac{1}{\ell} \quad B^{\prime \prime}=0$, except at $x=0$, and $\langle\beta\rangle=1 / 2(2 \ell)(1) / 2 \ell=1 / 2$. So,

$$
\mu_{1}<2 \frac{\sigma_{x}}{\ell}
$$

The second-order term in $\mu_{2}$ contributes only at $x=0$, which cannot be calculated by elementary means. If we approximate $B^{\prime \prime} \approx \frac{2}{\ell^{2}}$, then

$$
\mu_{2}=2 \frac{\sigma_{x}}{\ell}+\left(\frac{\sigma_{x}}{\ell}\right)^{2} \text {. }
$$

For $\beta=\cos ^{2} \alpha x, B^{\prime}=-\alpha \sin 2 \alpha x, B^{\prime \prime}=-2 \alpha^{2} \cos 2 \alpha x$,

and

$$
\mu_{1}=\frac{\pi}{\sqrt{2}} \frac{\sigma_{x}}{\ell} \approx 2.22 \frac{\sigma_{x}}{\ell} .
$$


In this case, there is no contribution from the second-order term, since the near-field profile is symmetric around $x=0$. Only asymmetric near fields will contribute here. In practice this will arise for steered beams.

Comparing eqs (23a) and (23b) one can see that the results are essentially model independent, since the constant coefficients are essentially equal and the other parameters enter exactly the same way.

On closer examination, it is found that for this example eq (19b) gives the most stringent condition on $\sigma_{x}$, i.e.,

$$
\sigma_{x}^{2} \ll \frac{1}{2}\left(\frac{l}{\pi}\right)^{2}
$$

must hold for the analysis presented in section 3 to be valid. If $L \equiv 2 l$ is the aperture dimension, then $\sigma_{x} \ll L / 9$. Let $L=n \lambda$, where $\lambda$ is the wavelength and $n$ is some constant, then $\sigma_{x} \ll m(0.1 \lambda)$, where $m>1$. In practice, such a root-mean-square position error is attainable. Using eq (22), one can write

or

$$
R(d B) \simeq 40 \frac{\sigma_{x}}{L}
$$

$$
\frac{\sigma_{x}}{\lambda} \simeq \frac{1}{40} n R \text {. }
$$

This last relationship gives the RMS displacement error in units of wavelengths in terms of $R$, the far-field error in $d B$, and $n$, the aperture size, in units of wavelength.

\subsection{Maximization of General Complex Near-Field Error Integral}

For general complex near fields or for errors in the off-axis direction, the expression whose amplitude is to be maximized has both real and imaginary parts; i.e.,

$$
I=\int G_{r}(x, \delta x(x)) d x+i \int G_{j}(x, \delta x(x)) d x .
$$

$G_{r}\left(G_{j}\right)$ is the real (imaginary) part of the integrand in the numerator of eq (1). One can either maximize the real (imaginary) part using the same 
procedure as for on-axis real near fields, but the maximum of the amplitude of I in eq (26) will not, in general, be thus attained. Only if

$$
\int g_{r} g_{j} d x=0
$$

where $g_{r}=\left[G_{r}\right]^{\prime}, g_{i}=\left[G_{i}\right]^{\prime},[Q]^{\prime}=\left.\frac{\partial Q}{\partial(d x)}\right|_{\delta x}=0$, will the maximum of $I$ be given by the larger of the maximum of the two integrals in eq (26). The proof of this simple fact will not be detailed here. If condition (27) does not hold, we look for the function that will maximize eq (26) as a linear combination of functions that maximize each of the integrals separately. Thus,

$$
\delta x=\alpha \delta x^{(r)}+\beta \delta x^{(i)}
$$

where $\delta x^{(r)}\left(\delta x^{(i)}\right)$ is the displacement error function which maximizes the real (imaginary) part of the (26), and $\alpha$ and $\beta$ are constants determined by conditions (31) and (32) below. Adapting the results in section 3, a firstorder approximation scheme to maximize eq (26) is as follows:

Let

$$
\begin{aligned}
& \delta x^{(r)}=g_{r} \\
& \delta x^{(i)}=g_{i} \\
& I_{r}=\int g_{r}^{2} d x \\
& I_{i}=\int g_{j}^{2} d x \\
& I_{m}=\int g_{r} g_{i} d x .
\end{aligned}
$$

Then expanding eq (26) for small $\delta x(x)$ and from eqs (11) and (28)

$$
\begin{gathered}
|I|^{2}=\left(\alpha I_{r}+\beta I_{m}\right)^{2}+\left(\alpha I_{m}+\beta I_{i}\right)^{2} \\
2 \ell \sigma_{x}^{2}=\alpha^{2} I_{r}+2 \alpha \beta I_{I n}+\beta^{2} I_{i}
\end{gathered}
$$

and one deterinines the parameters $\alpha$ and $\beta(\alpha)$ from

$$
\frac{d}{d a} L_{\alpha} L^{2}=0
$$




$$
\frac{d\left(\sigma_{x}^{2}\right)}{d a}=0
$$

The details of this calculation are presented in Appendix $A$, where final expression for $\alpha$ and $\beta$ are derived as well as conditions that must hold for the special cases $\alpha \neq 0, \beta=0$ and $\alpha=0, \beta \neq 0$ to maximize eq (29) subject to constraint (30).

A more detailed treatment of a specific case of eq (26) is given in section 3.4 and Appendix B.

3.3 Maximization for $\underline{k} \neq 0$

If in the region of interest the near field is real, the off-axis error displacement function

$$
\delta x=\frac{B^{\prime}(x)\left\{\begin{array}{ll}
\sin k x \\
\cos k x
\end{array}\right\}}{2 \eta-B^{\prime \prime}\left\{\begin{array}{l}
\sin k x \\
\cos k x
\end{array}\right\}}
$$

will maximize the integral

$$
\int B^{\prime}(x) \delta x\left\{\begin{array}{ll}
\sin k x \\
\cos k x
\end{array}\right\} d x
$$

where $n$ is determined by eq (11). If eq (27) holds, i.e.,

$$
\int\left[B^{\prime}(x)\right]^{2} \cos k x \sin k x d x=0
$$

either integral with sin $k x(\cos k x)$ will maximize the corresponding fractional error $\mu$. For near fields symmetric about the origin eq (35) will be satisfied. In practice, most near fields have a small asymmetric component, so eq (35) is only approximately satisfied. In case the asymmetry is significant, the procedure outlined in sections $3.2,3.4$ and in Appendix $B$ has to be followed. The results are for $|D(k)| \neq 0$

$$
\mu_{1}^{2} \equiv\left|\frac{\Delta D(k)}{D(k)}\right|^{2}\left\langle\sigma_{x}^{2} \frac{\left\langle B^{\prime} 2 \sin ^{2} k x\right\rangle}{\langle B(x) \cos k x\rangle^{2}+\langle B(x) \sin k x\rangle^{2}}\right.
$$


and

$$
\left|\frac{\Delta D(k)}{D(0)}\right|^{2} \leqslant \sigma_{x}^{2} \frac{\left\langle B^{\prime 2} \sin ^{2} k x\right\rangle}{\langle B(x)\rangle^{2}}
$$

and similarly for cos kx. Similar calculations to obtain second-order corrections could be easily performed.

In Appendix C, eq (37) is evaluated for the simple model $B(x)=\cos ^{2} \alpha x$ in figure 1 , and the results are compared to real simulations.

\subsection{Steered Beams}

In the case of steered beams, displacement errors both in the scan plane and perpendicular to the scan plane have components along the off-axis beam direction. One can model such a beam to zeroth order by the near field

$$
B(x)=b(x) e^{i \varepsilon x}
$$

where $\varepsilon$ is some wave number and $b(x)$ is one of the profiles depicted in figure 1. Mathematically, the problem of maximizing the error integral (either for $\underline{k}=\underline{\varepsilon}$, or $\underline{k} \neq \underline{\varepsilon}$ ) can be simplified if one keeps in mind the ratio in eq (5d); i.e., if the beam is steered enough off axis so that displacement errors in the scan plane correspond geometrically to displacement errors parallel to $\underline{k}$, with a sinall additional effect due to errors perpendicular to k. If the beam angle is $\theta$, a first-order approximation is

$$
\mu_{1} \approx c_{\|} \sigma_{\|}|\underline{\varepsilon}|\left\{\begin{array}{l}
\cos \theta \\
\sin \theta
\end{array}\right\}+c_{\perp} \frac{\sigma_{\perp}}{l}\left\{\begin{array}{l}
\sin \theta \\
\cos \theta
\end{array}\right\}
$$

where, depending on the magnitude of $\theta$, one of the terms is negligible compared to the other. The choice of the trigometric function depends on whether one is examining $z$ or $x-y$ displacement errors. For example, for $\theta=0$, on $7 y$ the term $c_{\|} \sigma_{\|}|\varepsilon|$ constributes for $z$ errors, and for $x-y$ errors only the term $c_{\perp} \sigma_{1} / \ell$ contributes. For $\theta=\pi / 2$ similar reasoning shows that the role of each term in eq (39) is interchanged. For angles such that any error has a significant projection both parallel and perpendicular to k, eq (39) is essentially valid, but the full analysis as outlined in section 3.2 and Appendix $A$ has to be carried out to determine the constants. 
For $\underline{k} \neq \underline{\varepsilon}$, the expressions in section 3.3 can be easily adopted for beams steered sufficiently off axis. One merely has to put the symbols in that section as

$$
\begin{gathered}
k+\varepsilon-k \\
B(x) \rightarrow b(x) \\
B^{\prime}(x) \rightarrow \varepsilon b(x)
\end{gathered}
$$

and

$$
k=0 \rightarrow k=\varepsilon \text {. }
$$

The adaption of the maximization procedure for steered beams is detailed in Appendix B.

4. The Error-Contaminated Far Field

The error-contaminated spectrum of the near field can be calculated exactly if the near field of the antenna and the exact displacement-error function are known. Let $D_{e}(\underline{K})$ be the error-contaminated far field,

$$
D_{e}(\underline{k})=\int B(\underline{x}+\delta \underline{x}(\underline{x})) e^{-i \underline{k} \cdot \underline{x}} d^{2} x
$$

which, for errors in the $x$-direction only, can be expanded as

$$
D_{e}(K)=\int\left[B(x)+\frac{\partial B}{\partial x}(x) \delta x+\frac{1}{2 !} \frac{\partial^{2}}{\partial x^{2}} B(x)(\delta x)^{2}+\cdots\right] e^{-i k \cdot x}-d^{2} x .
$$

The general expansion is a three-dimensional Taylor series. Since

$$
B(\underline{x})=\int D\left(\underline{K}^{\prime}\right) e^{\underline{i k^{\prime} \cdot \underline{x}} d^{2} K^{\prime}}
$$

spatial derivatives of $B(x)$ can be obtained from eq (42). Each differentiation will introduce a factor of $i k_{j}, j=1,2,3$ into the integrand of eq (41) with the result that

$$
D_{e}(\underline{k})=\int D\left(\underline{K}^{\prime}\right)\left[\int e^{i\left(\underline{k}^{\prime}-\underline{k}\right) \cdot \underline{x}} e^{i \underline{k} \cdot \delta \underline{x}(\underline{x})} d^{2} x\right] d^{2} K^{\prime}
$$

gives the error contaminated spectrum in terms of the known exact spectrum and the known displacement error function. Equation (43) can be written as 


$$
D_{\underline{e}}(\underline{K})=\int D\left(\underline{K}^{\prime}\right) E\left(\underline{K}^{\prime}, \underline{K}\right) d^{2} K^{\prime}
$$

where

$$
E\left(\underline{K}^{\prime}, \underline{K}\right)=\int e^{i\left(\underline{k}^{\prime}-\underline{k}\right) \cdot \underline{x}} e^{i \underline{k}^{\prime} \cdot \delta \underline{x}(\underline{x})} d^{2} x \text {. }
$$

If $\underline{\delta x}(\underline{x}) \equiv 0$, then $E\left(K^{\prime}, K\right)=\delta\left(\underline{k}^{\prime}-\underline{k}\right)$ and $D_{e}(\underline{K})=U(\underline{K})$; i.e., there is no error in the spectrum. If $\delta \underline{x}(\underline{x}) \equiv \underline{c}$, the result is essentially the same, as merely a phase factor $e^{i \underline{k} \cdot \underline{c}}$ is introduced. Another simple special case follows if $\delta \underline{x}(\underline{x})=\left(\alpha_{1} x, \alpha_{2} y\right)$ as discussed in [3].

For small $\delta \underline{x}(\underline{x})$ such that $\underline{k} \cdot \delta \underline{x} \ll 1$, eq (43) immediately yields a firstorder approximation

$$
D_{e}(\underline{K})=\int D\left(\underline{K}^{\prime}\right) E_{1}\left(\underline{K}^{\prime}, \underline{K}\right) d^{2} K^{\prime}
$$

where

$$
E_{1}\left(\underline{K}^{\prime}, \underline{K}\right)=\delta\left(\underline{K}-\underline{K}^{\prime}\right)+\int e^{i\left(\underline{k}^{\prime}-\underline{k}\right) \cdot \underline{x}} i \underline{k} \cdot \delta \underline{x} d^{2} x \cdot
$$

If we model $\delta x$ as a sum of delta functions, i.e., $\delta \underline{x}(\underline{x})=\sum_{-n} \delta\left(\underline{x}_{-}-\underline{x}_{-n}\right)$ where $\varepsilon_{n}$ is a complex amplitude and $x_{n}$ are the grid points ${ }^{n}$ where measurements are taken, then eq (44b) yields

$$
\Delta D(\underline{K}) \equiv D_{e}(K)-D(\underline{K})=i \underline{k} \cdot\left(\sum_{n} \varepsilon_{n} B\left(x_{n}\right) e^{-i \underline{k} \cdot \underline{x}-n}\right) \cdot
$$

Here, $\varepsilon_{n}$ is an unrestricted amplitude of the displacement function, and hence could be a complex random number. In such an event, eq (44c) gives the effects of random displacement errors on the far field.

\section{Displacement Errors in Spherical Scanning}

It has been observed experimentally that for electrically large antennas the near-field amplitudes obtained in planar and spherical scanning are essentially equal [3]. Thus,

$$
\left|B_{s}(\underline{r})\right|:\left|B_{p}(\underline{x})\right|
$$


where $B_{s}(r)$ is the spherical and $B_{p}(\underline{x})$ is the planar near field and' $\underline{r}$ is the general three-dimensional position vector. The phases across the main beam, however, differ, primarily due to the change in the $z$ displacement between the probe and the antenna. In a planar scan, the phase is essentially constant, but in the spherical scan the significant phase is given by

$$
\begin{gathered}
k \Delta z(x) \approx \pi\left(\frac{R}{\lambda}\right)\left(\frac{x}{R}\right)^{2}, y=0 \\
\left(\frac{x}{R}\right)<1
\end{gathered}
$$

where $R$ is the radius of the scan sphere and $x^{2}+z^{2}=R^{2}$ is the intersection of the scan sphere and the $y=0$ plane.

The simple expressions in eqs (45) and (46) can be exploited in spherical error analysis to take advantage of the results obtained in planar error analysis: one merely has to transform the phase of $B_{S}(r)$ according to eq (46) and approximate $B_{p}(\underline{x})$ with $B_{s}(\underline{r})$. The additional effect due to the variation of the orientation of the probe in spherical scanning as a function of position relative to the constant orientation of the probe in planar scanning is only significant at extrerne angles and is neglected in this section. Accordingly, errors in spherical scanning will, in general, be a linear combination of $z$ and $x-y$ errors in planar scanning. The linear coefficients will depend on an averaged geometric relationship between the sphere and the plane, as will now be shown.

Consider a displacement error function $\delta \underline{\theta}(\theta)$ along an arc that projects onto the $x$-and $z$-axis at $y=0$. The $x$ - and $z$-components of $\delta \theta$ are

or

$$
\delta \underline{\theta}(\theta)=\delta \theta(\theta)[(\hat{\theta} \cdot \hat{x}) \hat{x}+(\hat{\theta} \cdot \hat{z}) \hat{z}]
$$

$$
R \delta \underline{\theta}(\underline{\theta})=\delta \theta(\theta)[R \cos \theta \hat{x}-R \sin \theta \hat{z}] \text {. }
$$

In planar notation, eq (47) is

$$
\begin{gathered}
\delta x(x)=\delta \theta(x) R \cos \theta=z \delta \theta(x) \\
\delta z(x)=-\delta \theta(x) R \sin \theta=-x \delta \theta(x)
\end{gathered}
$$


where $\theta=\sin ^{-1} \frac{x}{R}$, and $R$ is the scan radius. Both displacement error functions in eq (48) must be taken into account in the maximization procedure, and section 3 must be altered accordingly.

The constraint (corresponding to eq (11)) is now written as

$$
\frac{1}{R \Delta \theta} \int_{L}(R \delta \theta)^{2} \frac{R d \theta}{d x} d x=\sigma_{\theta}^{2}
$$

where $d \theta / d x=1 / z$. The expression to be maximized is now

$$
\hat{I}=\int_{L}\{B(x+R \delta \underline{\theta}(x))-B(x)\} d x-\lambda R \int_{L} \frac{(R \delta \theta)^{2}}{z} d x
$$

which to first order is

$$
\hat{I}=\frac{1}{R} \int_{L}\left\{\frac{\partial B}{\partial x} z-\frac{\partial B}{\partial z} x\right\}(R \delta \theta) d x-\lambda R \int_{L} \frac{(R \delta \theta)^{2}}{z} d x
$$

The functional derivative of $\hat{I}$ in eq (51) with respect to $(R \delta \theta(x))$ will give the maximizing function similar to eq (15). Since $\frac{\partial B}{\partial z}=i \gamma B$, the integrand in eq (51) is complex, in general, and the procedure outlined in section 3.2 must be followed. Each term is maximized separately by

and

$$
R \delta \theta(x)=\frac{1}{2 \lambda}\left(\frac{z}{R}\right)^{2} \frac{\partial B}{\partial x}
$$

$$
\operatorname{R\delta } \theta(x)=\frac{1}{2 \lambda}\left(\frac{z}{R}\right)\left(\frac{x}{R}\right) \frac{\partial B}{\partial z}
$$

Hence,

$$
R \delta \theta(x)=\sigma_{\theta} \frac{\left(\frac{z}{R}\right)^{2} B_{x}(x)}{\left\langle\left(\frac{z}{R}\right)^{3}\left(B_{x}\right)^{2}\right\rangle_{\theta}^{1 / 2}}
$$

where $B_{x}=\frac{\partial B}{\partial x}$ and one defines $\langle Q\rangle_{\theta} \equiv \frac{1}{R \Delta \theta} \int_{L} Q d x$, where $Q$ is any quantity. Similarly, for $z$-displacements 


$$
R \delta \theta(x)=\sigma_{\theta} \frac{\left(\frac{z}{R}\right)\left(\frac{x}{R}\right) B_{z}(x)}{\left\langle\left(\frac{z}{R}\right)\left(\frac{x}{R}\right)^{2}\left(B_{z}\right)^{2}\right\rangle_{\theta}^{1 / 2}}
$$

The integrands in eq (51) become

and

$$
\hat{I}=\sigma_{\theta} R \Delta \theta\left\langle\left(\frac{Z}{R}\right)^{3}\left(B_{x}\right)^{2}\right\rangle_{\theta}^{1 / 2}
$$

$$
\hat{I}=\sigma_{\theta} R \Delta \theta\left\langle\left(\frac{z}{R}\right)\left(\frac{x}{R}\right)^{2}\left(B_{z}\right)^{2}\right\rangle_{\theta}^{1 / 2}
$$

and the corresponding on-axis fractional errors are

or

$$
\mu_{1}^{2}(0) \leqslant \sigma_{\theta}^{2} \frac{\left\langle\left(\frac{z}{R}\right)^{3}\left(B_{x}\right)^{2}\right\rangle}{\langle B(x)\rangle^{2}}
$$

$$
\mu_{1}^{2}(0) \leqslant \sigma_{\theta}^{2} \frac{\left\langle\left(\frac{z}{R}\right)\left(\frac{x}{R}\right)^{2}\left(B_{z}\right)^{2}\right\rangle}{\langle B(x)\rangle^{2}} .
$$

Expressions (55)-(58) can be compared to eqs (20)-(21). The presence of the geometric factors $\left(\frac{z}{R}\right)$ and $\left(\frac{x}{R}\right)$ in these new expressions merely reflect the fact that the magnitude of $\underline{\delta \theta}(x)$ is being optimized rather than the displacements in the $x-y$ plane.

Equations (52) and (58) individually maximize the respective terms in eq (51). However, as we have seen in section 3.2, the maximization of $|\hat{\mathrm{I}}|$ in eq (50) is given by the linear combination

$$
R \delta \theta(x)=\alpha\left(\frac{z}{R}\right)^{2} \frac{\partial B}{\partial x}+\beta\left(\frac{z}{R}\right)\left(\frac{x}{R}\right)\left|\frac{\partial B}{\partial z}\right|
$$

where $\alpha$ and $\beta$ are determined using the method outlined in section 3.2 and Appendix A.

The corresponding treatment for radial displacement errors is outlined in Appendix D. 


\subsection{A Simple Model in Spherical Geometry}

In general, the on-axis fractional errors are essentially (from eqs (57)(58))

$$
\mu_{1}^{2}(0) \leqslant c_{x}^{2}\left(\frac{\sigma \theta}{\ell}\right)^{2}\left(\frac{\bar{z}}{R}\right)^{3}+c_{z}^{2} \sigma_{\theta}^{2} k^{2}\left(\frac{\hat{z}}{R}\right)\left(\frac{\hat{x}}{R}\right)^{2}+c_{x z}^{2} \sigma_{\theta}^{2}\left(\frac{k}{l}\right)\left(\frac{\tilde{z}}{R}\right)^{2}\left(\frac{\tilde{x}}{R}\right)
$$

where $\tilde{z}, \bar{z}, \hat{z}, \hat{x}$ and $\tilde{x}$ are some intermediate values in the range $(0, R)$. For narrow-beam antennas $\ell \gg \lambda$, the second term predominates, but for very narrow beams, all the terms might be equally important. The simple models in figure 1 can be used to estimate in somewhat more detail the terms in eqs (57), (58) and (60). These estimates are, to leading order,

$$
\begin{aligned}
& \frac{3}{2}\left(\frac{\sigma}{R}\right)^{2} \frac{\theta_{\max }}{\sin ^{3} \theta_{\max }} \text { for } x \text {-component } \\
& \frac{1}{4} \sigma^{2} k^{2} \frac{\theta_{\max }}{\sin ^{3} \theta_{\max }} \text { for } z \text {-component }
\end{aligned}
$$

and

$$
\frac{4}{3} \sigma^{2} \frac{k}{\ell} \sin ^{3} \theta_{\max } \text { for the mixed component. }
$$

These expressions are comparable to eqs (5b) and (5c) and to (23) and (25).

\section{Summary}

The effects of probe displacement errors on the far-field spectrum have been examined both for planar and spherical scanning. Expressions for the displacement errors that maximize the error in the far-field have been derived using a method well known in the calculus of variations. The treatment of the planar case is straightforward, but the spherical problem is complicated by the fact that an error in a spherical coordinate corresponds to both $x-y$ and $z$ errors in planar geometry. Hence, a more complicated maximization procedure had to be adopted after the spherical data were transformed both in amplitude and phase onto the plane. To first order all fractional errors can be expressed as functions of $c \frac{\sigma}{\ell}$, where $c$ is some constant of order unity, $\ell$ is the relevant length scale either parallel or orthogonal to the direction in $k-$ space under observation, and $\sigma$ is an integral measure (constraint) of the total mean-square error of the measuring system. 
7. Acknowledgments

Numerous discussions with Allen C. Newell on the previous approaches to the subject area treated in this study are gratefully acknowledged. Useful interaction with Dr. David Hill and Dr. James Randa is also acknowledged.

8. References

[1] Kerns, D. M. Plane wave scattering-matrix theory of antennas and antenna-antenna interactions. Nat. Bur. Stand. (U.S.) Monogr. 162; 1981.

[2] Yaghjian, A. D. Upper-bound errors in far-field antenna parameters determined from planar near-field measurements. Part 1: Analysis. Nat. Bur. Stand. (U.S.) Tech. Note 667 ; 1975.

[3] Newel1, A. C. Upper-bound errors in far-field antenna parameters determined from planar near-field measurements. Part 2: Analys is and computer simulation. Lecture notes for National Bureau of Standards Short Course, Antenna Parameter Measurements by Near-Field Techniques: Boulder, Colorado; 1975. Private communication.

[4] Mathews, J.; Walker, R. L. Mathematical methods of physics, second edition. Massachusetts: W. A. Benjamin, Inc.; 1970. 501 p.

[5] Taylor, A. E. Advanced calculus. New York: Ginn and Company; 1955. 786 p.

[6] Conway, J. B. Functions of one complex variable, second edition. New York: Springer-Verlag; 1984. 320 p. 


\section{Appendix A}

Maxinization of General Complex Near-Field Error Integrals

Some Further Details

In section 3.2, the maximization of general complex near-field error integrals has been outlined. Here we present some important details. All quantitites used in this appendix have been previously defined in section 3.2 .

Condition (32) results in

$$
\frac{d \beta}{d \alpha}=-\frac{\alpha I_{r}+\beta I_{m}}{\alpha I_{m}+\beta I_{j}}
$$

and conditions (31) and (Ala) give

$$
\frac{d \beta}{d \alpha}=\frac{-I_{m}+\frac{d \beta}{d \alpha} I_{r}}{I_{i}-\frac{d \beta}{d \alpha} I_{m}}
$$

Equation (Ala) leads to

$$
\alpha=-\beta \frac{I_{m}+\frac{d \beta}{d \alpha} I_{i}}{I_{r}+\frac{d \beta}{d \alpha} I_{m}},
$$

and eq (Alb) leads to

$$
\left(\frac{d B}{d \alpha}\right)^{2}+\frac{d B}{d \alpha}\left[\frac{I_{r}-I_{i}}{I_{m}}\right]-1=0
$$

and since the discriminant $b^{2}-4 a c>0$ one obtains real solutions, with $I \equiv \frac{I_{r}-I_{i}}{2 I_{m}}$,

$$
\left(\frac{d \beta}{d \alpha}\right)_{ \pm}=-\bar{I} \pm \sqrt{\bar{I}^{2}+1} \text {. }
$$

The choice of the sign in eq $(A 3)$ is arbitrary. $B_{ \pm}^{\prime} \equiv\left(\frac{d B}{d \alpha}\right)_{ \pm}$can then be substituted into eq $(A 2 a)$ to obtain $\alpha$ in terms of $B$, $\bar{i} . e$. , 


$$
\alpha_{ \pm}=-\beta \frac{I_{m}+\beta_{ \pm}^{\prime} I_{j}}{I_{r}+\beta_{ \pm}^{T} I_{m}} \equiv-\beta f_{ \pm}
$$

which can be used to obtain $\beta$ from eq (30):

$$
\beta_{ \pm}=\sigma \sqrt{2 l}\left(f_{ \pm}^{2} I_{r}-2 f_{ \pm m} I_{m}+I_{j}\right)^{-1 / 2} \text {. }
$$

Equations (A4) and (A5) provide the coefficients in the displacement error function (28) that maximizes the general error integral (26).

Treating the special case $\alpha \neq 0, \beta=0$, that corresponds to maximizing only the real part of eq (26), one obtains from (A2a)

$$
\frac{d \beta}{d \alpha}=-\frac{I_{r}}{I_{m}}
$$

and from $(A 1 D)$

$$
\left[\int g_{r} g_{j} d x\right]^{2}-\left[\int g_{r}^{2} d x\right]^{2}=0
$$

which is not, in general, satisfied. For example, $g_{r}=g_{j}$ would satisfy eq (A7). Similar results hold for $\alpha=0, \beta \neq 0$, i.e., one must have

$$
\left[\int g_{r} g_{j} d x\right]^{2}-\int g_{r}^{2} d x \int g_{j}^{2} d x=0
$$

This shows that these special cases do not maximize, in general, the amplitude of the integral in eq (26). 
Appendix B

The Maximization Procedure for Steered Beams

In this appendix the qualitative physical treatment presented in section 3.4 is made more precise. Only the essential details are presented.

For steered beams, from section 3.4 ,

$$
B(x)=b(x) e^{i \varepsilon x}
$$

The error spectrum is then given by

$$
\Delta D(k)=\int\left[b(x+\delta x) e^{i \varepsilon(x+\delta x)}-b(x) e^{j \varepsilon x}\right] e^{-i k x} d x
$$

where $\delta x(x)$ is subject to constraint (11). The method presented in section 3 can be adapted to yield a condition for $\delta x(x)$ that will maximize the real (imaginary) part of eq (B1). For $k=\varepsilon$, one obtains for the real part

$$
\delta x=\frac{1}{2 \lambda}\left[b^{\prime}(x+\delta x) \cos (\varepsilon \delta x)-\varepsilon b(x+\delta x) \sin (\varepsilon \delta x)\right]
$$

corresponding to eq $(10 c)$. For small $\varepsilon \delta x$, the zeroth-order approximation immediately follows; i.e., let $\cos (\varepsilon \delta x) \approx 1, \sin (\varepsilon \delta x) \approx 0$, and eq $(10 c)$ is recovered. Higher order approximations are obtained by expanding in eq (B2) the near-field quantities and the trigonometric functions in Taylor and infinite series, respectively, and collecting terms in increasing powers of $\delta x$. Thus,

$$
\delta x=\frac{1}{2 \lambda}\left[b^{\prime}(x)+\left[b^{\prime \prime}(x)-\varepsilon^{2} b(x)\right] \delta x+\frac{1}{2}\left[b^{\prime \prime \prime}(x)-\varepsilon^{2} b^{\prime}\right](\delta x)^{2}+\cdots\right]
$$

This expression should be compared to the expansion above eq (12). To first order then,

$$
\delta x=\frac{b^{\prime}(x)}{2 \lambda-\left[b^{\prime \prime}(x)-\varepsilon^{2} b(x)\right]}
$$




$$
b^{\prime \prime}(x)-\varepsilon^{2} b(x)<2 \lambda(1)
$$

where the supercript indicates first order, then the constraint (11) gives

$$
\delta x \simeq \sigma_{x} \frac{b^{\prime}(x)}{\left\langle b^{\prime 2}\right\rangle} 1_{/ 2}
$$

or

$$
2 \lambda^{(1)}=\frac{1}{\sigma_{x}}\left\langle b^{\prime} 2\right\rangle^{1 / 2}
$$

Again, for small enough $\sigma_{x}$ condition (B5) can be satisfied, as well as conditions corresponding to eqs (19a) and (19b). The second-order maximization of the real part of eq $(B 1)$, using $(B 6)$, is

$$
\mu_{2}(\varepsilon) \leqslant \frac{1}{\langle b\rangle}\left[\sigma_{x}\left\langle b^{\prime 2}\right\rangle^{1 / 2}+\frac{1}{2} \sigma_{x}^{2} \frac{\left\langle\left(b^{\prime \prime}-\varepsilon^{2} b\right) b^{\prime 2}\right\rangle}{\left\langle b^{\prime 2}\right\rangle}\right] .
$$

This expression corresponds to eq (22).

The imaginary part of eq (B1) can be maximized similarly to the above procedure. One obtains

$$
\delta x=\frac{1}{2 \lambda}\left[b^{\prime}(x+\delta x) \sin (\varepsilon \delta x)+\varepsilon b(x+\delta x) \cos (\varepsilon \delta x)\right]
$$

and

$$
\delta x \cong \frac{\varepsilon b(x)}{2 \lambda-\varepsilon b^{\top}(x)}
$$

where, if $2 \lambda \gg \varepsilon b^{\prime}(x)$, then from the constraint (11),

$$
2 \lambda=\frac{\varepsilon}{\sigma_{x}}\left\langle b^{2}\right\rangle \frac{1 / 2}{2}
$$

and

$$
\delta x \stackrel{\sim}{=} \frac{b(x)}{x\left\langle b^{2}\right\rangle / 2}
$$

The second-order maximization of the imaginary part of eq (B1), using $(B 12)$, is 


$$
\mu_{2}(\varepsilon)\left\langle\frac{\varepsilon}{\langle b\rangle}\left[\sigma_{x}\left\langle b^{2}\right\rangle \frac{1 / 2}{\sigma^{2}}{ }^{2} \frac{\left\langle b^{2} b^{2}\right\rangle}{\left\langle b^{2}\right\rangle}\right] .\right.
$$

To maximize the amplitude of $\Delta D(\varepsilon)$, we construct the linear combination

$$
\delta x=a b(x)+\beta b^{\prime}(x)
$$

and use the method outlined in section 3.2 and Appendix $A$ to solve for $\alpha$ and B. This will not be presented here. Finally, the case $k \neq \varepsilon$ could be fully developed exactly along the lines presented in this appendix. 


\section{Appendix $C$}

The Far-Field Error Spectrum

In this appendix the theoretical result in section 3.3 is evaluated for the model near field $B(x)=\cos ^{2} \alpha x$, where $2 \alpha \equiv k^{\prime}=\frac{\pi}{\ell}$. Equation (37) gives the imaginary part of the fractional error, whereas the real part is given by

$$
\left|\frac{\Delta D(k)}{D(0)}\right|^{2}\left\langle\sigma_{x}^{2} \frac{\left\langle B^{\prime} 2 \cos ^{2} k x\right\rangle}{\langle B(x)\rangle^{2}}\right.
$$

When the averages are evaluated one obtains

$$
\left|\frac{\Delta D(k)}{D(0)}\right|^{2}<\sigma_{x}^{2} \alpha^{2}\left(1 \pm \operatorname{sincq} \mp \operatorname{sinc}\left(q^{\prime}-q\right) \mp \operatorname{sinc}\left(q^{\prime}+q\right)\right)
$$

where $\sin c(q)=\sin (q) / q, q=2 k \ell$ and $q^{\prime}=2 k^{\prime} \ell=2 \pi$, and the upper (1ower) set of signs give the real (imaginary) part of the fractional error, respectively. These functions of $k$ have been plotted in figure $\mathrm{C}-1$.

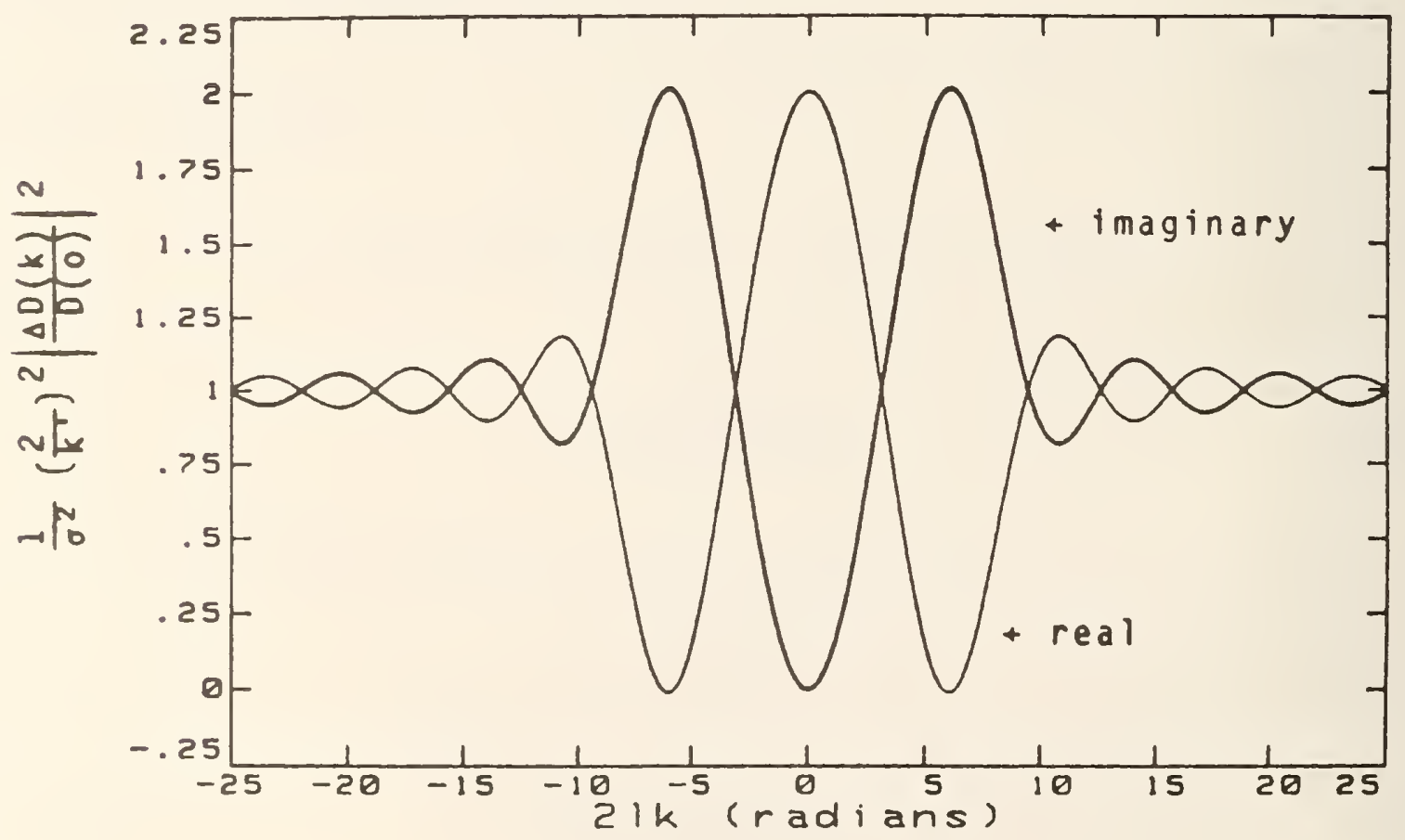

Figure $C-1$. The amplitude squared of the normalized maximum fractional error as a function of $k$ for a near field $B(x)=\cos ^{2} \alpha \times 2 \alpha \equiv k^{\prime}=\pi / \ell$. 
Using the error displacement function that gives a maximum error on-axis, the fractional error spectrum as a function of $k$ is given by

$$
\frac{\nabla D(k)}{D_{0}}=\frac{{ }^{\sigma} x}{\langle B\rangle\left\langle B^{\prime 2}\right\rangle^{1 / 2}} \cdot\left[\int_{-\ell}^{\ell} B^{\prime 2} \cos k x d x+i \int_{-\ell}^{\ell} B^{\prime 2} \operatorname{sink} x d x\right] .
$$

When this is evaluated for $B(x)=\cos ^{2} \alpha x$, one obtains

$$
\left|\frac{\nabla D(k)}{D(0)}\right|^{2}=2^{3} \quad \sigma_{x}^{2} a^{2}\left[\frac{1}{2} \operatorname{sinc} \frac{q}{2}-\frac{1}{4} \operatorname{sinc}\left(q^{\prime}+\frac{q}{2}\right)-\frac{1}{4} \operatorname{sinc}\left(q^{\prime}-\frac{q}{2}\right)\right]^{2}
$$

where $q\left(q^{\prime}\right)$ have been defined previously.

In figure $\mathrm{C}-2 \mathrm{a}$ the effects of experimentally induced near field errors on the far field are shown. The maximum error occurs on axis. In figure $c-2 b$ the expression ( $C 4$ ) is plotted. The qualitative agreement between the experimental and theoretical curves is apparent. For a general (realistic) near field, the error spectrum will be given by a sum over $k$ ' of functions given in eq (C2) wherein each term is weighted by the spectral component of the square of the derivative of the near field, as can be seen in eqs (37) and (C1).

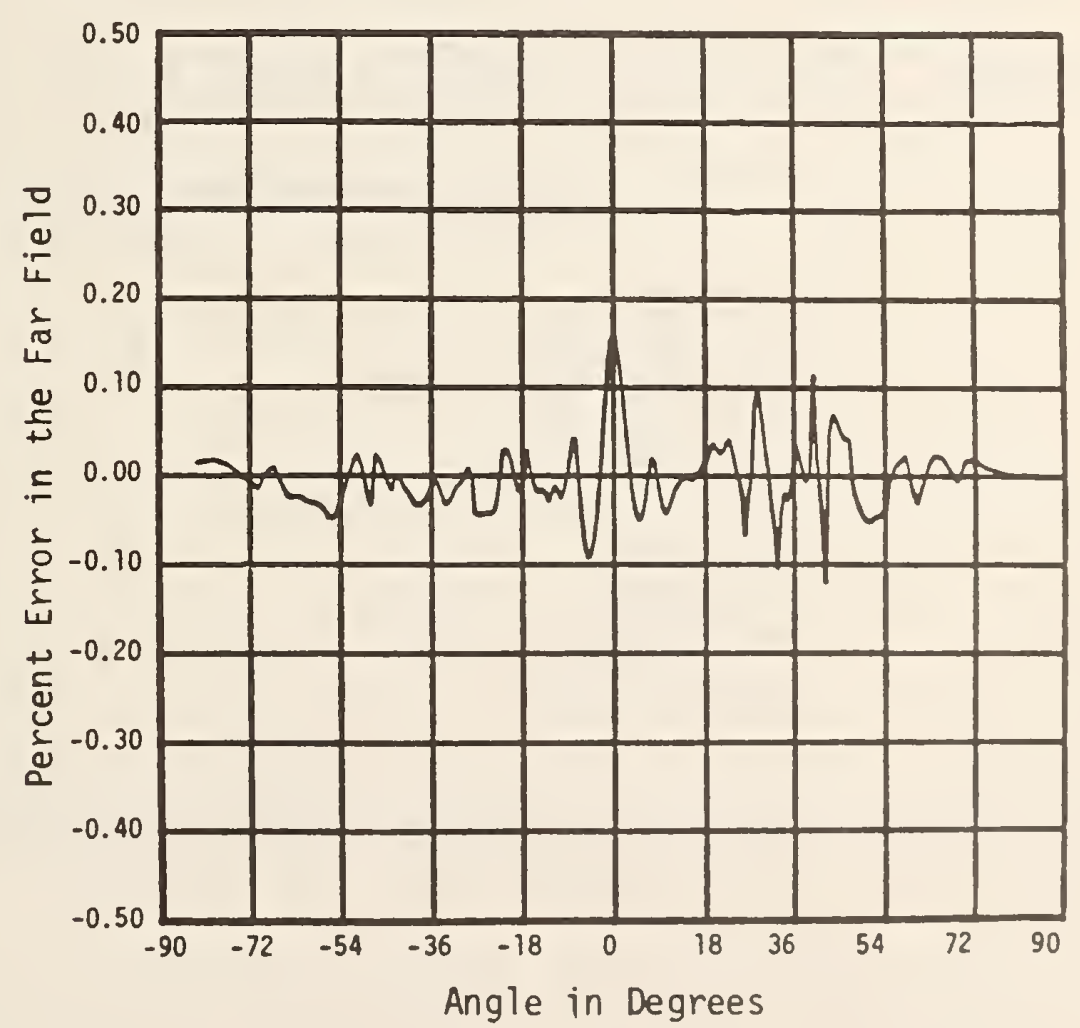

Figure $\mathrm{C}-2 \mathrm{a}$. Error in percent of maximum amplitude in far-field pattern due to $x$-position error (same as figure 17 in [3]). 


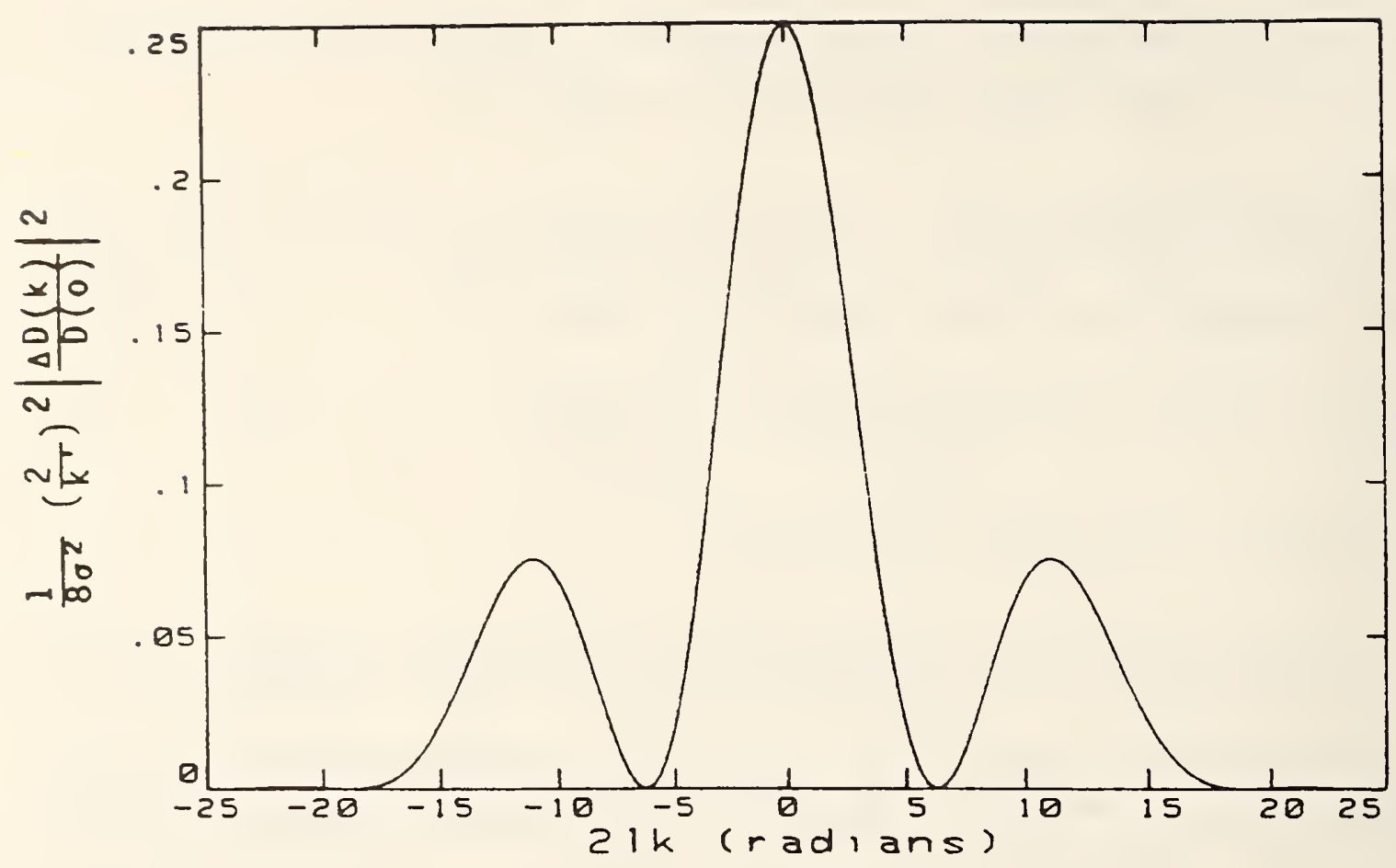

Figure $\mathrm{c}-2 \mathrm{~b}$. Theoretical fractional error in the spectrum when the on-axis error is the maximum. 


\section{Appendix D}

\section{Radial Displacement Errors in Spherical Scanning}

In this appendix the equations analyzing $\theta$-displacement errors in section 5 are adapted to displacement errors in the radial direction, denoted by $\delta r(\theta)$. The error displacement function is

$$
\delta \underline{r}(\theta)=\delta r(\theta)\left[\left(\frac{x}{R}\right) \hat{x}+\left(\frac{z}{R}\right) \hat{z}\right],
$$

corresponding to eq (48), and the equation of constraint is

$$
\frac{1}{R \Delta \theta} \int_{L}(\delta r)^{2} \frac{R d \theta}{d x} d x
$$

corresponding to eq (49). The expression to be maximized is

$$
\hat{I}=\int_{L}\{B(x+\delta r)-B(x)\} d x-\lambda R \int_{L} \frac{(\delta r)^{2}}{z} d x
$$

or

$$
\left.\hat{I}=\int\left\{\frac{\partial B}{\partial x}\left(\frac{x}{R}\right)+\frac{\partial B}{\partial z}\left(\frac{Z}{R}\right)\right\} \delta r(x) d x-\lambda R \int_{L} \frac{(\delta r}{z}\right)^{2} d x
$$

Each term is maximized individually by

$$
\delta r(x)=\frac{\sigma_{r}\left(\frac{x}{R}\right)\left(\frac{z}{R}\right) B_{x}(x)}{\left\langle\left(\frac{z}{R}\right)\left(\frac{x}{R}\right)^{2}\left(B_{x}\right)^{2}\right\rangle_{\theta}}
$$

and

$$
\delta r(x)=\frac{\sigma_{r}\left(\frac{z}{R}\right)^{2} B_{z}(x)}{\left\langle\left(\frac{z}{R}\right)^{3}\left(B_{z}\right)^{2}\right\rangle_{\theta}}
$$

corresponding to eqs (53) and (54). The on-axis fractional errors are given by 


$$
\mu_{1}^{2}(0) \leqslant \sigma_{r}^{2} \frac{\left\langle\left(\frac{z}{R}\right)\left(\frac{x}{R}\right)^{2}\left(B_{x}\right)^{2}\right\rangle}{\langle B(x)\rangle^{2}},
$$

and

$$
\mu_{1}^{2}(0) \leqslant \sigma_{r}^{2} \frac{\left\langle\left(\frac{z}{R}\right)^{3}\left(B_{z}\right)^{2}\right\rangle}{\langle B(x)\rangle^{2}} .
$$

The amplitude of $\hat{\mathrm{I}}$ is maximized by a linear combination of eqs (D5) and (06), and an expression similar to eqs (60) and (61) can be written immediately. 


\begin{tabular}{|c|c|c|c|}
\hline U.S. DEPT. OF COMM. & 1. PUBLICATION OR & 2. Performing Organ. Report No. & 3. Publication Date \\
\hline $\begin{array}{l}\text { BIBLIOGRAPHIC DATA } \\
\text { SHEET (See in structions) }\end{array}$ & NBS/TiN- 1306 & & October 1986 \\
\hline
\end{tabular}

4. TITLE AND SUBTITLE

Displacement Errors in Antenna Near-Field Measurements and The ir Effect on the Far Field

5. AUTHOR(S)

Lorant A. Muth

6. PERFORMING ORGANIZATION (If joint or other than NBS, see instructions)

7. Contract Grant No.

NATIONAL BUREAU OF STANDARDS

DEPARTMENT OF COMMERCE

WASHINGTON, D.C. 20234

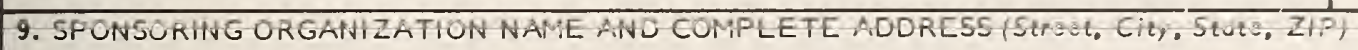

8. Type of Report \& Period Covered

10. SUPPLEMENTARY NOTES

[.] Document describes a computer program; SF-185, FIPS Software Summary, is attached.

11. ABSTRACT (A 200-word or less factual summary of most significant information. If document includes a significant bibliography or literature survey. mention it here)

The effects of probe displacement errors in the near-field measurement procedure on the far-field spectrum are studied. Expressions are derived for the displacement error functions that maximize the fractional error in the spectrum both for the on-axis and off-axis directions. Planar $x-y$ and $z$-displacement errors are studied first and, consequently, the results are generalized to errors in spherical scanning. Some simple near-field models are used to obtain order of magnitude estimates for the fractional error as a function of relevant scale lengths of the near field, defined as the lengths over which significant variations occur.

12. KEY WORDS (Six to twelve entries; alphabetical order: capitalize only proper names; and separate key words by semicolons) error maximization; far-field spectrum; near fields; off-axis fractional errors; on-axis fractional errors; planar scanning; probe displacement errors; spherical scanning

13. AVAILABILITY

[X] Unlimited

$\square$ For Official Distribution. Do Not Release to NTIS

$[X]$ Order From Superintendent of Documents, U.S. Government Printing Office, Washington, D.C. 20402.

$[$ Order From National Technical Information Service (NTIS), Springfield, VA. 22I6I
14. NO. OF PRINTED PAGES

36

15. Price 



\section{Periodical}

Journal of Research-The Journal of Research of the National Bureau of Standards reports NBS research and development in those disciplines of the physical and engineering sciences in which the Bureau is active. These include physics, chemistry, engineering, mathematics, and computer sciences. Papers cover a broad range of subjects, with major emphasis on measurement methodology and the basic technology underlying standardization. Also included from time to time are survey articles on topics closely related to the Bureau's technical and scientific programs. Issued six times a year.

\section{Nonperiodicals}

Monographs-Major contributions to the technical literature on various subjects related to the Bureau's scientific and technical activities.

Handbooks-Recommended codes of engineering and industrial practice (including safety codes) developed in cooperation with interested industries, professional organizations, and regulatory bodies.

Special Publications-Include proceedings of conferences sponsored by NBS, NBS annual reports, and other special publications appropriate to this grouping such as wall charts, pocket cards, and bibliographies.

Applied Mathematics Series-Mathematical tables, manuals, and studies of special interest to physicists, engineers, chemists, biologists, mathematicians, computer programmers, and others engaged in scientific and technical work.

National Standard Reference Data Series-Provides quantitative data on the physical and chemical properties of materials, compiled from the world's literature and critically evaluated. Developed under a worldwide program coordinated by NBS under the authority of the National Standard Data Act (Public Law 90-396).

NOTE: The Journal of Physical and Chemical Reference Data (JPCRD) is published quarterly for NBS by the American Chemical Society (ACS) and the American Institute of Physics (AIP). Subscriptions, reprints, and supplements are available from ACS, 1155 Sixteenth St., NW, Washington, DC 20056.

Building Science Series-Disseminates technical information developed at the Bureau on building materials, components, systems, and whole structures. The series presents research results, test methods, and performance criteria related to the structural and environmental functions and the durability and safety characteristics of building elements and systems.

Technical Notes-Studies or reports which are complete in themselves but restrictive in their treatment of a subject. Analogous to monographs but not so comprehensive in scope or definitive in treatment of the subject area. Often serve as a vehicle for final reports of work performed at NBS under the sponsorship of other government agencies.

Voluntary Product Standards-Developed under procedures published by the Department of Commerce in Part 10, Title 15, of the Code of Federal Regulations. The standards establish nationally recognized requirements for products, and provide all concerned interests with a basis for common understanding of the characteristics of the products. NBS administers this program as a supplement to the activities of the private sector standardizing organizations.

Consumer Information Series-Practical information, based on NBS research and experience, covering areas of interest to the consumer. Easily understandable language and illustrations provide useful background knowledge for shopping in today's technological marketplace.

Order the above NBS publications from: Superintendent of Documents, Government Printing Office, Washington, DC 20402.

Order the following NBS publications-FIPS and NBSIR's-from the National Technical Information Service, Springfield, VA 22161.

Federal Information Processing Standards Publications (FIPS PUB)-Publications in this series collectively constitute the Federal Information Processing Standards Register. The Register serves as the of ficial source of information in the Federal Government regarding standards issued by NBS pursuant to the Federal Property and Administrative Services Act of 1949 as amended, Public Law 89-306 (79 Stat. 1127), and as implenented by Executive Order 11717 (38 FR 12315, dated May 11, 1973) and Part 6 of Title 15 CFR (Code of Federal Regulations).

NBS Interagency Reports (NBSIR)-A special series of interim or linal reports on work performed by NBS for outside sponsors (both government and non-government). In general, initial distribution is handled by the sponsor; public distribution is by the National Technical Information Service, Springfield, VA 22161, in paper copy or microfiche form. 
U.S. Department of Commerce National Bureau of Standards

Gaithersburg. MD 20899

Official Business

Penalty for Private Use $\$ 300$ 